\title{
Safety and Quality of Care for Seniors Living with Dementia
}

Alexey Dudevich, Liudmila Husak, Tracy Johnson and Allie Chen

\begin{abstract}
The number of Canadian seniors living with dementia is increasing because of the aging population and population growth. This article highlights various aspects of safety and quality of care for seniors with dementia as described in the Dementia in Canada digital report.

In particular, it was found that seniors with dementia are more likely to fall and suffer fall-related injuries than seniors without dementia. Similarly, seniors with dementia are more likely to experience unintended harm during their hospital stay, such as urinary tract infections, dehydration and pneumonia. In long-term care, seniors with dementia were found to be at higher risk of being physically restrained and given potentially inappropriate antipsychotic drugs than other seniors. These results can inform current and future efforts to improve the safety and quality of care for Canadian seniors with dementia.
\end{abstract}

\section{Introduction}

In Canada, the number of seniors living with Alzheimer's disease and other forms of dementia is rising steadily, thus creating a growing demand on healthcare systems across the country. In 2013-2014, an estimated 402,000 seniors were living with dementia, with approximately 76,000 new cases of dementia diagnosed in Canada every year (Public Health Agency of Canada 2017). Almost two-thirds of seniors with dementia reside in the community; the remaining one-third reside in long-term care and nursing homes (Canadian Institute for Health Information 2018b).

This article highlights different aspects of safety and quality of care for seniors with dementia as described in the Dementia in Canada digital report (Canadian Institute for Health Information 2018b). It includes analysis of falls experienced by seniors across different care sectors, explores the use of antipsychotics and restraints in long-term care and provides a snapshot of hospital harm pertaining to hospital stays. Please refer to the Dementia in Canada digital report for further information and research findings related to dementia: https://www.cihi.ca/en/ dementia-in-canada.

\section{Seniors and Falls}

The Canadian Institute for Health Information (CIHI) finds that seniors with dementia have higher rates of falls than seniors without dementia. In particular, it was found that:

- $16 \%$ of seniors with dementia who live in a long-term care facility have fallen in the past 30 days, compared to $11 \%$ of seniors without dementia;

- $15 \%$ of emergency department visits by seniors with dementia are fall related, compared to $9 \%$ by seniors without dementia;

- $16 \%$ of hospital admissions by seniors with dementia are fall related, compared to $7 \%$ by seniors without dementia; and

- among seniors who receive home care, rates of falls are similarly high in those with dementia (39\%) and those without $(37 \%)$.

The proportion of seniors with dementia in long-term care or nursing homes who had fallen in the past 30 days was 1.5 times higher than the proportion of seniors without dementia. In three provinces that submit data to $\mathrm{CIHI}$, there was moderate variation in falls over the five years from 2011 to 2015, with a slight increase in fall rates among all senior residents, particularly in Ontario and Alberta (Figure 1).

Among seniors receiving homecare services, 39\% with dementia had a fall in the 90 days before their assessment, similar to the percentage of seniors without dementia (37\%). Men with dementia receiving home care fell more (42\%) than women $(37 \%)$. There was a substantial provincial variation in the percentage of homecare clients with dementia with a record of falls. Homecare programs in Ontario and British Columbia (BC) reported that $40 \%$ of clients fell, compared to $30 \%$ in Alberta. These percentages represent unadjusted rates. Fall prevention programs and safe practices targeting those receiving homecare services should be a priority because of the high rates of falls. 
FIGURE 1.

Proportion of seniors who fell in the last $\mathbf{3 0}$ days in long-term care (unadjusted rates)

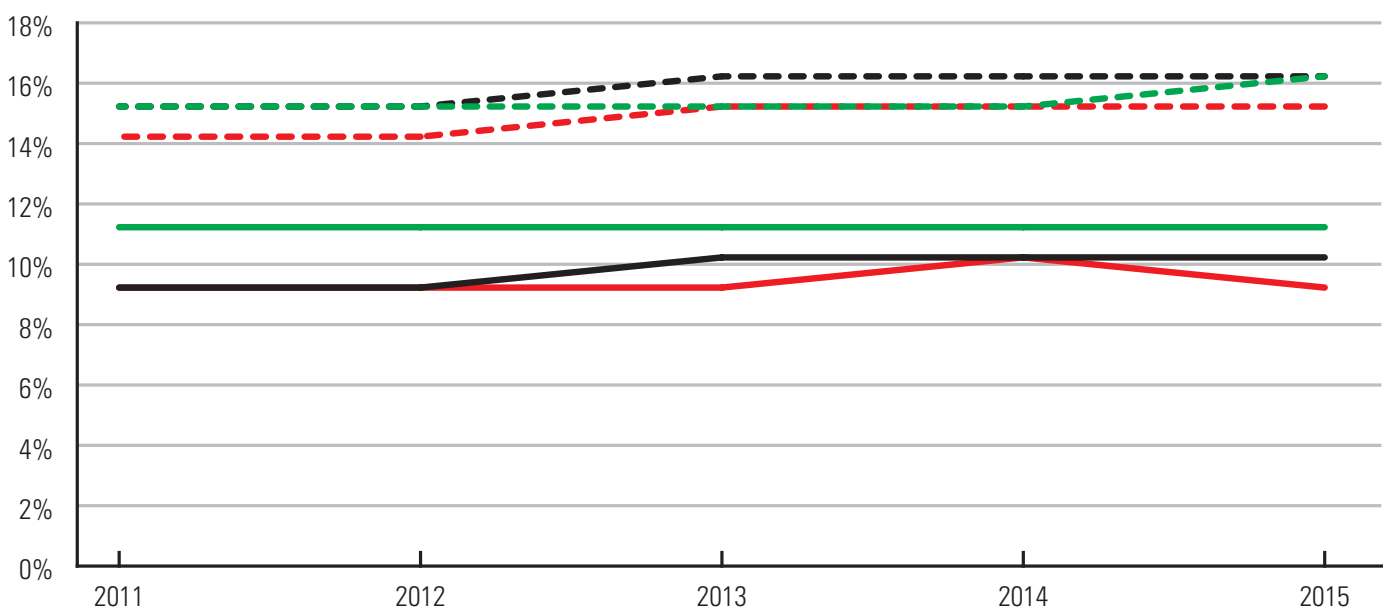

Ontario

- - With dementia Without dementia

Alberta

-..- With dementia

Without dementia

British Columbia

-.- With dementia Without dementia

FIGURE 2.

Potentially inappropriate antipsychotic drug use among seniors in long-term care, percentage, 2011-2012 to 2015-2016 (unadjusted)

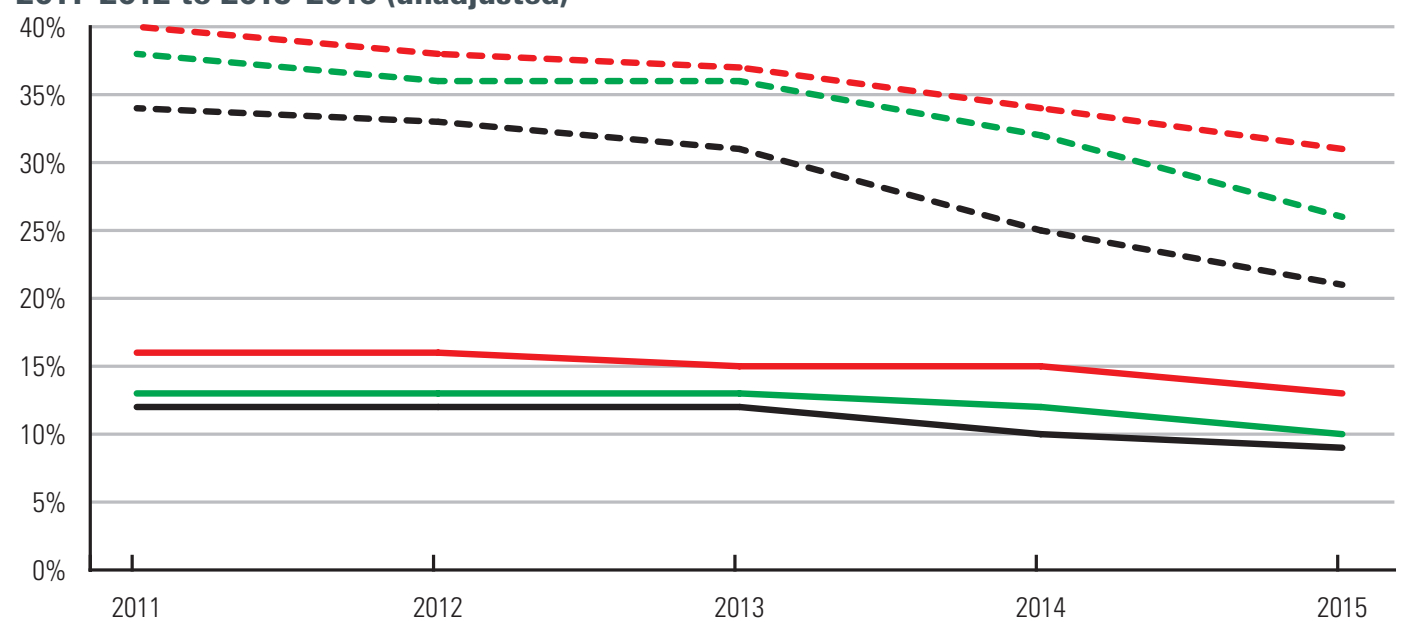

FIGURE 3.

Restraint use among seniors in long-term care, percentage, 2011-2012 to 2015-2016 (unadjusted)

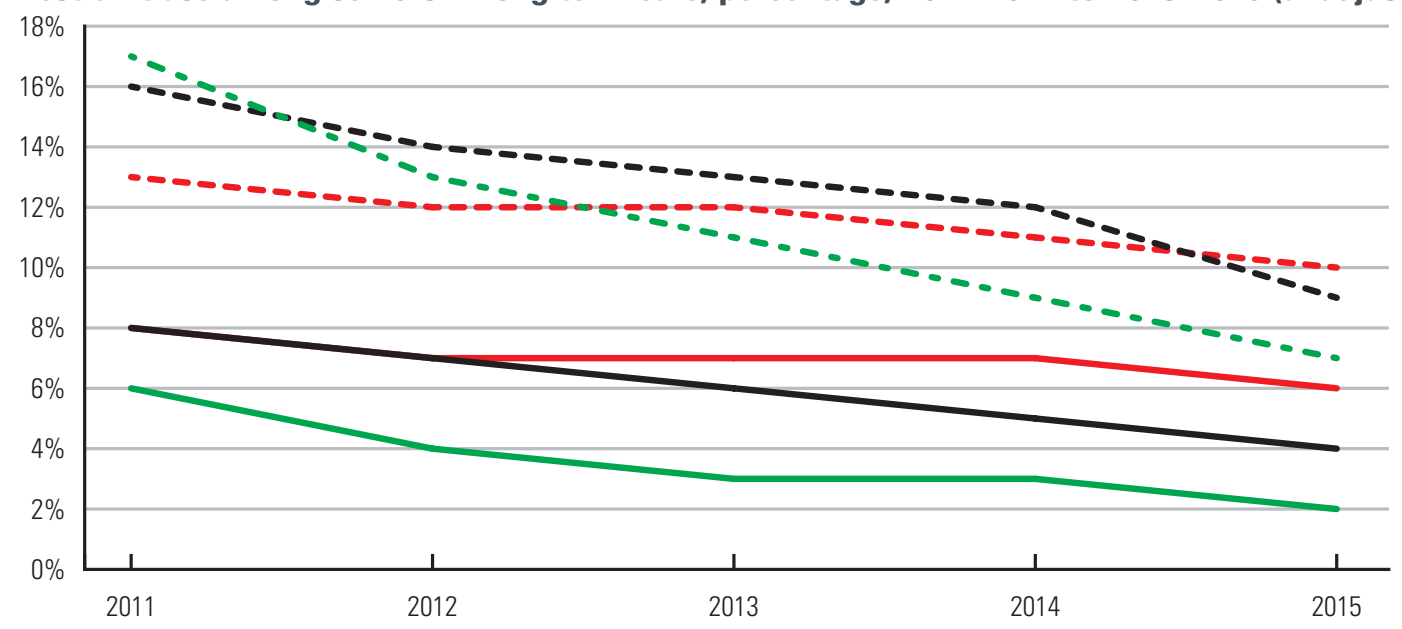

Figures 1-3 from Continuing Care Reporting System, (CIHI 2018a) 


\section{Use of Antipsychotics and Restraints in Long-Term Care}

In long-term care, Canadian seniors with dementia are at a higher risk of being physically restrained and given potentially inappropriate antipsychotic drugs than seniors without dementia. However, policy changes and culture change programs in long-term care homes have helped spur a decrease in this trend over the past several years.

Potentially inappropriate antipsychotic drug use among seniors with dementia decreased between 2011-2012 and $2015-2016$ from $38 \%$ to $26 \%$ in Ontario, $34 \%$ to $21 \%$ in Alberta and $40 \%$ to $31 \%$ in BC. This decrease was more pronounced among seniors with dementia than those without (Figure 2).

The use of physical restraints (such as wheelchair lap belts or bed rails) also decreased over the same period. Between 2011 and 2015 , it dropped from $17 \%$ to $7 \%$ in Ontario, $16 \%$ to $9 \%$ in Alberta and $13 \%$ to $10 \%$ in BC. (Figure 3.)

\section{Seniors and Hospital Harm}

Hospital harm occurs more often among seniors with dementia than among those without dementia. Hospital harm is defined as a hospitalization with at least one unintended occurrence of harm that could be potentially prevented by implementing known evidence-informed practices (Canadian Institute for Health Information and Canadian Patient Safety Institute 2016; Canadian Institute for Health Information 2018c). In 2015-2016, $11.5 \%$ of seniors with dementia experienced hospital harm, compared to $7.9 \%$ of seniors without. The leading types of harm were urinary tract infections $(3.5 \%)$, dehydration $(2.6 \%)$, pneumonia $(2.5 \%)$ and delirium (2.3\%).

Longer length of stay was the strongest predictor of hospital harm - seniors with dementia who stayed in hospital for more than a week had seven times higher odds of experiencing hospital harm compared with seniors without dementia (odds ratio $=7.0,95 \%$ confidence interval $=6.5-7.4$ ).

CIHI analysis shows that seniors with dementia stayed in hospital much longer than those without; their median length of stay was 1.3-2 times higher. Among those age 65-79 years, the median length of stay for seniors with dementia was eight days, compared to four days for seniors without. The gap decreased among those age $\geq 80$ : eight days for seniors with dementia and six days for seniors without.

The findings suggest that the risk of hospital harm may be reduced if unnecessary hospitalizations for seniors with dementia are limited and lengths of stay are shortened.

\section{Way Forward}

Efforts are currently under way to improve the safety and quality of care for Canadian seniors with dementia. Sharing experiences from notable interventions may provide insights and inspiration for further work in this area, as in the examples provided below.

Ontario's Long-Term Care Homes Act requires long-term care homes to reduce the use of restraints (both chemical and physical) wherever possible. In response, Behavioural Supports Ontario launched a team-based program in 2010 to enhance healthcare services for Ontario's seniors, their caregivers and their families (Grouchy et al. 2017).

In Alberta, the Appropriate Use of Antipsychotics Initiative informed and was reinforced by amendments to the Continuing Care Health Service Standards (CCHSS). The 2016 CCHSS requirement for monthly reviews of antipsychotics used as restraints is key to enabling antipsychotic reductions (Alberta Health Services 2018).

The Canadian Patient Safety Institute's Safer Healthcare Now! implemented the Reducing Falls and Injuries from Falls Intervention (Safer Healthcare Now! 2015), which includes:

- the Falls Prevention/Injury Reduction Getting Started Kit, a guide to help professionals working across health sectors implement fall prevention/injury reduction programs for people age $\geq 65$ years;

- a series of national patient safety webinars that disseminate emerging evidence and help stimulate knowledge transfer; and

- a measurement system to assess progress toward improvement goals.

These and other initiatives help to ensure that health systems continue to meet the needs of seniors with dementia, particularly as their numbers grow as a result of aging of the Canadian population. $\mathrm{HQ}$

\section{References}

Alberta Health Services. 2018. Appropriate Use of Antipsychotics (AUA) Toolkit. Retrieved May 17, 2018. <https://www.albertahealthservices. $\mathrm{ca} /$ scns/auatoolkit.aspx>.

Canadian Institute for Health Information. 2018a. Continuing Care Metadata. Retrieved July 30, 2018. <https://www.cihi.ca/en/ continuing-care-metadata>.

Canadian Institute for Health Information. 2018b. Dementia in Canada. Retrieved July 30, 2018. <https://www.cihi.ca/en/dementiain-canada>.

Canadian Institute for Health Information. 2018c. Hospital Harm. Retrieved July 30, 2018. <http://indicatorlibrary.cihi.ca/display/ HSPIL/Hospital+Harm>. 
Canadian Institute for Health Information and Canadian Patient Safety Institute. 2016. "Measuring Patient Harm in Canadian Hospitals." What Can Be Done to Improve Patient Safety? authored by Chan B., Cochrane D. Retrieved July 30, 2018. <https://secure.cihi.ca/free_products/cihi_ cpsi_hospital_harm_en.pdf>.

Grouchy, M., N. Cooper and T. Wong. 2017. "Implementation of Behavioral Supports Ontario (BSO): An Evaluation of Three Models of Care." Healthcare Quarterly 19(4): 39-73. doi: 10.12927/ hcq.2017.25013.

Public Health Agency of Canada. 2017. Dementia in Canada, including Alzheimer's Disease. Retrieved February 13, 2018. <https://www. canada.ca/en/public-health/services/publications/diseases-conditions/ dementia-highlights-canadian-chronic-disease-surveillance.html>.

Safer Healthcare Now! 2015. Reducing Falls and Injuries from Falls Getting Started Kit. Retrieved July 30, 2018. <http://www. patientsafetyinstitute.ca/en/toolsResources/Documents/Interventions/ Reducing\%20Falls\%20and\%20Injury\%20from \%20Falls/Falls\%20 Getting\%20Started\%20Kit.pdf >.

\section{About the Authors}

Alexey Dudevich, MPA, is a senior analyst in Health Reports at CIHI in Toronto, Ontario. He is responsible for performing analyses, providing methodological support to projects and writing reports.

Liudmila Husak, MD, MPH, is the manager of Health Reports at $\mathrm{CIHI}$ in Toronto, Ontario. She oversees the development and production of a variety of CIHI's analytical reports.

Tracy Johnson is the director of Health System Analysis and Emerging Issues at $\mathrm{CIHI}$ in Toronto, Ontario.

Allie Chen, MSc, is a senior analyst in Health Reports at CIHI, in Toronto, Ontario. She is responsible for performing analyses and providing methodological support to projects.

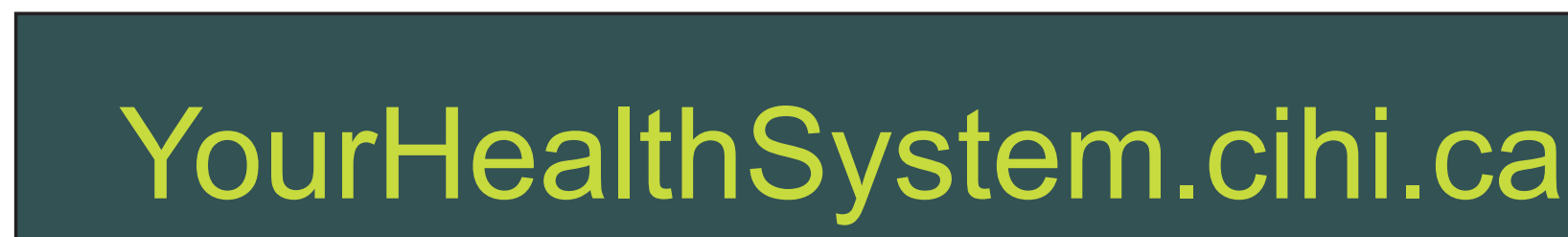

Providing health system decision-makers and Canadians with current data on indicators that are important to them

\begin{tabular}{|llll|}
\hline Your Health System: & $\vdots$ & Your Health System: & Your Health System: \\
& $\vdots$ & $\vdots$ & $\vdots$
\end{tabular}

